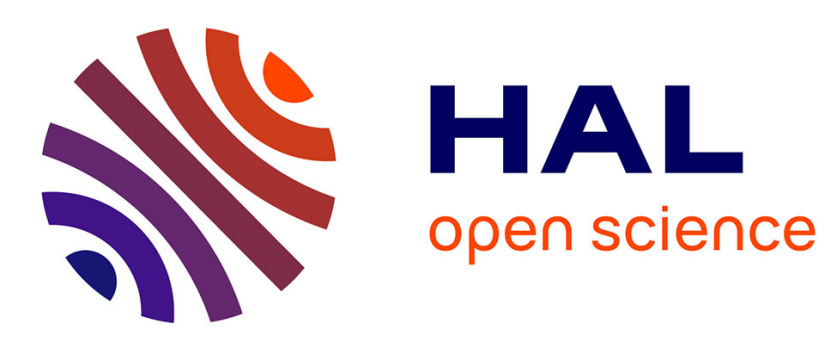

\title{
Chronique de droit pénal et de procédure pénale
} Évelyne Bonis-Garçon, Virginie Peltier

\section{To cite this version:}

Évelyne Bonis-Garçon, Virginie Peltier. Chronique de droit pénal et de procédure pénale. Nouveaux cahiers du Conseil constitutionnel, 2015, 48, pp.177-194. 10.3917/nccc1.048.0177 . hal-03472688

\section{HAL Id: hal-03472688 \\ https://hal.science/hal-03472688}

Submitted on 15 Dec 2021

HAL is a multi-disciplinary open access archive for the deposit and dissemination of scientific research documents, whether they are published or not. The documents may come from teaching and research institutions in France or abroad, or from public or private research centers.
L'archive ouverte pluridisciplinaire HAL, est destinée au dépôt et à la diffusion de documents scientifiques de niveau recherche, publiés ou non, émanant des établissements d'enseignement et de recherche français ou étrangers, des laboratoires publics ou privés. 


\section{Chronique de droit pénal et de procédure pénale}

Évelyne BONIS-GARCON Professeur agrégé

Université de Bordeaux Institut de sciences criminelles et de la justice (EA 4633)

\section{Virginie PELTIER}

Maître de conférences Université de Bordeaux Institut de sciences criminelles et de la justice (EA 4633)

\section{I - DROIT PÉNAL}

Agression sexuelle. Élément constitutif. Circonstances aggravantes

\section{Cons. const., 6 fév. 2015, ${ }^{\circ}$ 2014-448 QPC}

Le conseil était saisi d'une question prioritaire de constitutionnalité portant sur les articles 222-22 du code pénal - qui définit l'agression sexuelle comme toute atteinte sexuelle commise avec violence, contrainte, menace ou surprise - et 222-22-1 du même code précisant que la contrainte peut être physique ou morale, cette dernière pouvant résulter de la différence d'âge existant entre une victime mineure et l'auteur des faits et de l'autorité de droit ou de fait que celui-ci exerce sur cette victime. L'auteur de la question faisait valoir qu'en prévoyant qu'un élément constitutif du délit d'agression sexuelle puisse résul-ter de l'autorité de droit ou de fait que l'auteur des faits exerce sur la victime, alors que cette même autorité de droit ou de fait constitue, en vertu du $2^{\circ}$ de l'article 222-30 du code pénal, une circonstance aggravante de ce délit, ces dis-positions méconnaissaient le principe de légalité des délits et des peines.

Ce faisant, il tentait de se fonder sur la jurisprudence de la Cour de cassation selon laquelle un même élément de fait ne peut à la fois constituer un élé-ment constitutif de l'infraction et sa circonstance aggravante. Ainsi, à plusieurs reprises, la chambre criminelle avait-elle censuré des décisions de juges du fond qui, pour caractériser contrainte ou surprise, se fondaient sur le jeune âge de la victime ou l'autorité de fait ou de droit exercée par l'auteur des faits (Cass. crim., ler mars 1995, Bull. crim. $\mathrm{n}^{\circ} 92$; 21 oct. 1998, Bull. crim. n 274 : D. 1999, 75, obs. Mayaud, JCP G 1998, II, 10125, note Mayer, Dr. pén. 1998, comm. 5, obs. Véron ; 10 mai 2001, Bull. crim. n 116 : Dr. pén. 2001, 110, obs. Véron, Rev. sc. crim. 2001, 808, obs. Mayaud ; 14 nov. 2001 : Bull. crim. $\mathrm{n}^{\circ} 239$ ). La Cour de cassation reprochait plus précisément aux juges de ne pas caractériser concrètement la contrainte et, pour ce faire, de ne s'en tenir abs-traitement qu'à la mention du jeune âge des victimes ou de l'autorité de fait ou de droit exercée sur elles par leur agresseur. 
Cette situation était mal vécue par les victimes comme par les associations défendant leurs intérêts qui soulignaient que faire la preuve de la contrainte, donc de l'absence de consentement, était quasiment impossible quand l'atteinte se produisait dans le cadre familial avec la conséquence que les agressions étaient presque toujours traitées comme de simples atteintes sexuelles (infraction constituée sans la nécessité d'une contrainte, surprise, violence ou menace). Même si la chambre criminelle a par la suite infléchi sa position, retenant que la contrainte pouvait résulter du très jeune âge de la victime (Cass. crim., 7 déc. 2005, Bull. crim. n 326 : D. 2006, 1655, obs. Garé, AJ pénal 2006, 81, Dr. pén. 2006, comm. 31, obs. Véron, Rev. sc. crim. 2006, 319, obs. Mayaud, Rev. pénit. 2006, p. 152, note Malabat), la loi n 2010-121 du 8 février 2010 tendant à inscrire l'inceste commis sur les mineurs dans le code pénal a malgré tout eu alors la volonté de mettre fin à la situation en y introduisant un article 222-22-1 précisant la notion de contrainte. Seulement, le résultat n'est pas à la hauteur des souhaits puisque si les travaux parlemen-taires font état de la volonté législative de faire en sorte qu' « avec la rédaction proposée, la quasi-totalité des actes incestueux commis sur un mineur (soient) réputés commis sous l'emprise d'une contrainte et (soient) qualifiés en viol ou agression sexuelle » (rapport $\mathrm{n}^{\circ}$ 1601, M. L. Fort), la disposition ne répute rien, pas plus qu'elle n'établit de présomption de contrainte puisqu'elle pré-cise que la contrainte morale peut résulter (et non résulte) de la différence d'âge et de l'autorité de fait ou de droit. Cela révèle que le législateur, ne maîtrisant pas les distinctions juridiques, pourrait bien avoir voulu répondre à un problème de fond (un même fait ne peut être à la fois élément constitutif et circonstance aggravante de l'infraction) par une pseudo règle de preuve (une présomption qui, lecture faite, n'apparaît pas dans le texte). D'ailleurs, les critiques qu'il a adressées à la jurisprudence de la Cour de cassation le confirment : qu'un même fait ne puisse servir à la fois d'élément constitutif d'une infraction et de circonstance aggravante est une solution inhérente à la notion de circonstance aggravante : il serait pour le moins incohérent et injuste de retenir un élément de fait afin de considérer que l'infraction est constituée puis d'utiliser ce même élément pour aggraver la sanction norma-lement encourue... La Cour de cassation ne faisait donc que suivre un raisonnement imparable, même s'il n'était pas toujours compris par les victimes et ceux qui les défendent (voir, par exemple, en matière de récidive, Cass. crim., 21 oct. 2008 : Bull. crim. n²11).

En introduisant ces précisions sur la contrainte, le législateur n'a pas pour autant clarifié la situation puisque son innovation entendait seulement faciliter la preuve de la contrainte en la rendant « automatique » (l'acte devant être réputé commis sous l'emprise de la contrainte) du seul fait de la différence d'âge et du lien d'autorité entre agresseur et victime. Pis, en réclamant, pour illustrer la contrainte, une différence d'âge entre auteur et victime et l'autorité de droit ou de fait du premier sur la seconde, il a exclu de la protection qu'il voulait instaurer tous les enfants qui ne seraient pas liés à leur agresseur par une autorité de droit ou de fait (par exemple, le texte ne peut servir à carac-tériser la contrainte si les faits ont été commis par le grand frère de 30 ans sur la camarade de sa demi-sœur, âgée de quatre ans car il n'a pas autorité...). Certes, le texte se borne à donner une indication (" la contrainte morale peut résulter... ») mais on est alors en droit de s'interroger sur l'utilité de vouloir cumuler les critères de l'âge et de l'autorité et, en définitive, sur l'utilité du texte lui-même. C'est donc ces incohérences qui ont pu inciter l'auteur de la question à se prévaloir de la jurisprudence de la Cour de cassation pour tenter de le faire invalider. 
Le Conseil constitutionnel ne s'y est pas laissé prendre puisqu'il replace le débat sur son véritable terrain, précisant que 1'article 222-22-1 du code pénal ne définit pas un élément constitutif de l'agression sexuelle en apportant des précisions sur la contrainte, mais « a pour seul objet de désigner certaines circonstances de fait sur lesquelles la juridiction saisie peut se fonder pour apprécier si, en l'espèce, les agissements dénoncés ont été commis avec contrainte ». La conséquence qu'il en tire est logique : les articles 222-22 et 222-22-1 du code pénal ne définissent pas à la fois un élément constitutif et une circonstance aggravante de l'agression sexuelle, de sorte qu'ils ne portent pas atteinte au principe de la légalité des délits et des peines. Notons enfin, à titre de conclusion, que la chambre criminelle a rendu, le 18 février 2015 (Cass. crim., 18 fév. 2015, n 14-80.772) un arrêt par lequel elle rejette le pourvoi fait par un professeur de karaté, condamné, entre autres, pour agressions sexuelles aggravées sur de jeunes garçons. Elle estime que les juges de la chambre des appels correctionnels ont suffisamment caractérisé la contrainte morale en faisant référence au statut et à l'aura de professeur renommé du condamné qui lui conféraient une autorité certaine sur ses élèves, qu'il avait de surcroît placés dans une situation de dette en leur offrant des cadeaux. Et la cour de conclure que la contrainte résulte de l'autorité de fait qu'il exerçait sur eux. Or, cette affirmation lapidaire respire à son tour l'ambivalence car elle peut vouloir signifier, soit, qu'en l'espèce, l'autorité de fait du professeur, telle que concrètement caractérisée par les juges (cf. la décision du conseil : « en l'espèce »), impliquait la contrainte, soit que le luxe de détails fournis par la cour d'appel était inutile dans la mesure où, de toute façon, la contrainte résulte - de façon abstraite cette fois - de l'autorité de droit ou de fait de l'agresseur. Dans ce dernier cas, elle se placerait en contradiction totale avec la décision du conseil comme avec la volonté législative devant l'absence de clarté de laquelle celui-ci a tenté de composer. (V.P.)

\section{II - PROCÉDURE PÉNALE}

\section{1 - Audition libre. Loi dans le temps}

Cass. crim., 17 févr. 2015, QPC (non-lieu à renvoi)

L'arrêt rendu par la chambre criminelle de la Cour de cassation est l'occasion de revenir sur la question des effets dans le temps des lois et des décisions du Conseil constitutionnel à propos de l'audition libre.

Du point de vue normatif, on se souvient que jusqu'à récemment, le code de procédure pénale se bornait à prévoir qu'une personne pouvait être entendue au stade policier sans contrainte sans que la loi qui a créé ce statut, ne l'ait assorti de droits particuliers. C'est le Conseil constitutionnel qui, par une décision du 18 novembre 2011, est venu consacrer le statut de l'audition libre en prévoyant qu'une personne à l'encontre de laquelle il apparaît, avant son audition, ou au cours de celle-ci, qu'il existe des raisons plausibles de soupçonner qu'elle a com-mis ou tenté de commettre une infraction pour laquelle elle pourrait être placée en garde à vue, ne puisse être entendue ou continuer à être entendue libre-ment par les enquêteurs que si elle a été informée de la nature et de la date de l'infraction qu'on la soupçonne d'avoir commise et de son droit de quitter à tout moment les locaux de police ou de gendarmerie (Cons. const., 18 nov. 2011, QPC n ○ 2011/191/194/195/196/197). 
Liant ainsi ces droits à l'existence de soupçons et non à la situation de contrainte dans laquelle la personne ne se trouve pas placée (à la différence de la personne gardée à vue), le Conseil constitutionnel a vu sa solution reprise et amplifiée plus récemment par la loi n 2014-535 du 27 mai 2014 portant transposition de la directive 2012/13/UE du Parlement européen et du Conseil du 22 mai 2012 relative au droit à l'in-formation dans le cadre des procédures pénales. Désormais en effet, l'article 61-1 du code de procédure pénale, entré en vigueur le 2 juin 2014 pour la plupart de ses dispositions (voire au $1^{\text {er }}$ janvier 2015 pour les mentions relatives à l'assistance par avocat) reprend ces droits et les complète définissant ainsi un véritable statut de la personne en audition libre, à défaut d'un statut plus général de suspect (E. Vergès, Le statut juridique du suspect : un premier défi pour la transposition du droit de l'Union européenne en procédure pénale : Rev. Droit pénal 2014, étude 15). De cette évolution législative, un requérant entendait obtenir, si ce n'est le bénéfice par la remise en cause d'une procédure suivie contre lui sous l'empire de la loi ancienne, au moins un nouvel examen de la constitutionnalité de l'article 62 du code de procédure pénale dans sa rédaction antérieure à la loi. Il soumettait ainsi à la Cour de cassation une question prioritaire ainsi rédigée : "Les dispositions de l'article 62 du code de procédure pénale, dans leur rédaction anté-rieure à la loi du 27 mai 2014 transposant la directive 2012/13/UE du Parlement européen et du Conseil du 22 mai 2012 relative au droit à l'information dans le cadre des procédures pénales, en ce qu'elles ne prévoient pas l'assistance d'un avocat pour la per-sonne entendue librement qui est informée par les enquêteurs de la nature et de la date de l'infraction qu'on la soupçonne d'avoir commise, subordonnant ainsi le droit à l'assistance d'un conseil à l'existence d'une mesure de contrainte et non à la suspicion qui pèse sur la personne interrogée, portent-elles atteinte aux droits et libertés que la Constitution garantit et, plus exactement, aux droits de la défense garantis par l'article 16 de la Déclaration des droits de l'homme et du citoyen ». La critique était assurément pertinente au regard du droit européen et de la différence curieusement établie par le législateur entre la personne privée de liberté qui, pour cette raison avait des droits, et celle simplement soupçonnée qui n'en avait pas alors même que la défense ne se conçoit qu'en raison de soupçons et non en raison de la contrainte (sur ce point sur le fondement de l'article 6 de la Convention EDH, not. Cour EDH Serves c/ France, 20 oct. 1997, nº 82/1996/671/893 et Brusco c/ France, 14 janv. 2011, nº 1466/07). Elle ne pouvait en revanche recevoir les faveurs de la chambre criminelle qui, de façon constante, limite les droits aux personnes gardées à vue ou faisant l'objet d'une mesure de rétention douanière (v. en ce sens, not. Cass. crim., 3 avril 2013, pourvoi n ${ }^{\circ} 11-87333$; AJ pénal 2013, p. 411, obs. Ascenci, Rev. sc. crim. 2013, p. 842, obs. Salvat, Dr. pénal 2013, comm. 82, note Maron et Haas, RPDP 2014, p. 983 et s.). Pas plus, elle n'avait de chance de prospérer au regard de la jurisprudence du Conseil constitutionnel qui n'a opéré qu'une reconnaissance a minima de droits au profit de la personne en audition libre sans prévoir le droit à l'assistance d'un avocat (Cons. const., 18 nov. 2011, op. cit.).

Sans surprise, la Cour de cassation refuse de transmettre cette question au Conseil constitutionnel au motif que le texte applicable à la procédure étant l'article 62 dans son ancienne rédaction, il doit être interprété à l'aune de la jurisprudence alors en vigueur, à savoir selon les réserves d'interprétation émises en 2011 par le Conseil constitutionnel. La loi du 27 mai 2014 est sans effet sur les réserves d'interprétation émises en 2011 par le Conseil constitutionnel. 
La loi nouvelle du 27 mai 2014 ne saurait constituer un changement de circonstances au sens de l'article 23-2 de l'ordonnance du 7 novembre 1958.

2 - Détention provisoire. Délai de décision de la chambre de l'instruction

C. constit., 29 janvier 2015, déc. $n^{\circ} 2014-446$ QPC

$\mathrm{Si}$, pour les besoins de l'instruction, la privation de liberté avant jugement est possible, elle doit être justifiée et strictement limitée aux nécessités de la pro-cédure. Dès lors, le législateur a défini avec précision la durée ainsi que les motifs du placement en détention provisoire. Il a aussi exigé des juridictions qui peuvent être saisies de contestations relatives à ces mesures de statuer dans des délais pré définis afin que le droit à un recours effectif ne s'exerce pas au détriment de la liberté la plus fondamentale de chacun qu'est la liberté d'aller et venir. En ce sens, l'article 194, dernier alinéa du code de procédure pénale dispose : "En matière de détention provisoire, la chambre de l'instruction doit se prononcer dans les plus brefs délais et au plus tard dans les dix jours de l'appel lorsqu'il s'agit d'une ordonnance de placement en détention et dans les quinze jours dans les autres cas, faute de quoi la personne concernée est mise d'office en liberté, sauf si des vérifications concernant sa demande ont été ordonnées ou si des circonstances imprévisibles et insurmontables mettent obstacle au jugement de l'affaire dans le délai prévu au présent article ». En définissant de tels délais, le législateur a entendu veiller à la célérité dans le traitement du contentieux de la détention provisoire et à la préservation de la liberté de la personne mise en examen, laquelle est toujours, à ce stade de la procédure, présumée innocente. De façon comparable aux règles régissant l'appel contre l'ordonnance du juge des libertés et de la détention statuant en matière de détention provisoire, il est prévu qu'en cas de pourvoi en cassation contre la décision de la chambre de l'instruction, la chambre criminelle dispose d'un délai de trois mois à compter de la réception du dossier pour rendre sa décision, faute de quoi la personne mise en examen est mise d'office en liberté (Cpp. art. 567-2). Pourtant, cette fixation légale de délai pour statuer semble contenir une faille puisqu'aucune disposition similaire n'impose des délais pour statuer à la chambre de l'instruction lorsqu'elle est saisie sur renvoi de la Cour de cassation. Dans ce cas en effet, ne faut-il pas craindre qu'une personne soit maintenue en détention alors même que la décision qui fonde ce placement a pu être annulée par la Cour de cassation?

Avant de répondre à cette question et de comprendre l'enjeu de la décision du Conseil constitutionnel, il conviendra de rappeler les conséquences sur la liberté du pourvoi et de l'arrêt de la Cour de cassation. En effet, en matière de déten-tion provisoire, le pourvoi n'a pas d'effet suspensif de telle sorte que lorsqu'il est formé contre un arrêt confirmant une ordonnance de placement en détention provisoire ou refusant une mise en liberté, il ne fait pas obstacle à la détention de la personne. Pas plus, l'arrêt de la chambre criminelle venant annuler un tel arrêt n'emporte la mise en liberté de la personne qui demeure détenue en vertu du titre décerné par le juge et de la détention. Dès lors, en n'enfermant pas la chambre de l'instruction dans un délai pour statuer sur renvoi de la Cour de cassation, ne faut-il pas craindre que la détention ne se prolonge abusivement? 
C'est précisément au regard de ce risque que la chambre criminelle de la Cour de cassation avait transmis au Conseil constitutionnel par un arrêt du 12 novembre 2014, la question prioritaire de constitutionnalité d'un requérant se prévalant du fait que l'absence de texte imposant à la chambre de l'instruction, lorsqu'elle est saisie sur renvoi après cassation d'un arrêt rejetant l'appel formé contre une ordonnance de placement en détention provisoire, de statuer dans un délai déterminé, serait contraire à la fois au principe d'égalité, au droit au respect de la présomption d'innocence et au droit à la liberté individuelle tel qu'il découle de l'article 66 de la Constitution. La Cour de cassation avait estimé la question sérieuse au motif que «la personne mise en examen se trouve de ce fait dans l'impos-sibilité de connaître le délai dans lequel sera examinée la légalité de la décision et de faire sanctionner le dépassement de ce délai » (Cass. crim., 12 nov. 2014, n 14-86016, inédit).

Ainsi saisi, le Conseil constitutionnel a d'abord fait le constat d'un silence du code de procédure pénale de telle sorte qu'il s'agissait pour lui moins de s'inter-roger sur la conformité d'une loi à la constitution que de la jurisprudence de la Cour de cassation qui, de façon constante, relève-t-il (déc. consid. $\mathrm{n}^{\circ} 3$ ), juge que la chambre de l'instruction saisie sur renvoi n'est pas tenue de se prononcer dans les délais prévus à l'article 194 alinéa $4 \mathrm{du}$ code, lequel n'est applicable que dans le cas où cette juridiction statue " initialement» soit lors d'un appel contre une ordonnance du juge des libertés et de la détention (v. en ce sens, Cass. crim., 21 nov. 1969: Bull. crim. n 311 ; Rev. sc. crim.1969, p. 421, obs. J. Robert ; plus récemment Cass. crim., 10 avril 2002, pourvoi $\mathrm{n}^{\circ}$ 02-80886 ; 23 févr. 2010, pourvoi $\mathrm{n}^{\circ} 09-88068$; 24 mai 2011, pourvoi $\mathrm{n}^{\circ} 11-81118$ ).

Il a ensuite envisagé cet état du droit au regard de deux principes fondamentaux : d'une part, les principes constitutionnels de respect de la liberté individuelle et de la présomption d'innocence et d'autre part, le principe d'égalité devant les règles de procédure pénale.

Sur le premier point, la décision du Conseil constitutionnel mérite la plus grande atteinte car s'il conclut au fait que l'absence de disposition législative fixant un délai maximum dans lequel la chambre de l'instruction doit statuer lorsqu'elle est saisie en matière de détention provisoire sur renvoi de la Cour de cassation ne porte pas atteinte aux exigences constitutionnelles s'est après avoir formulée une importante réserve d'interprétation. Il indique en effet "qu'en matière de privation de liberté, le droit à un recours juridictionnel effectif impose que le juge judiciaire soit tenu de statuer dans les plus brefs délais ». Après avoir ainsi rattaché la célérité dans le traitement contentieux au droit à un recours juridictionnel effectif tel qu'il se dégage de l'article 16 de la Déclaration des droits de l'homme et du citoyen, le Conseil précise « qu'il appartient aux autorités judiciaires, sous le contrôle de la Cour de cassation, de veiller au respect de cette exigence y compris lorsque la chambre de l'instruction statue sur renvoi de la Cour de cassation ». Il résulte donc de cette décision que même en l'absence de délais légaux s'imposant à la chambre de l'instruction pour statuer sur renvoi de la Cour de cassation, une obligation de célérité s'impose à elle. De ce point de vue, la décision du Conseil n'est pas sans rappeler la jurisprudence de la chambre criminelle de la Cour de cassation qui juge de façon constante, une fois qu'elle a fait le constat de l'inapplicabilité du texte définissant 
un délai pour statuer en appel au cas d'un renvoi après cassation, que la cour d'appel « n'a d'autre devoir que de statuer au plus tôt » (Cass. crim., 21 nov. 1968 : op. cit.) ou qu'elle est tenue de se prononcer « dans un bref délai» (Cass. crim., 24 mai 2011, op. cit.).

Cette solution permet certes de protéger le détenu mais au prix d'une situa-tion procédurale assez complexe pour lui. La présence d'un délai est en effet très avantageuse dans la mesure où, en principe, le fait pour le juge de ne pas avoir statué dans le délai imparti a pour conséquence obligatoire une levée de la mesure privative de liberté. En revanche, en l'absence de délai pour statuer, il incombe au détenu qui estimerait que la décision de la cour de renvoi tarde à intervenir, de saisir le juge d'instruction d'une demande de mise en liberté comme l'autorise à le faire, à tout moment de la procédure, l'article 148 du code (v. le rappel de cette procédure par le conseil, consid. $\mathrm{n}^{\circ}$ 7).

Même si rien ne l'impose au plan constitutionnel, l'intervention d'une modi-fication textuelle imposant un délai pour statuer offrirait ainsi une solution plus confortable au détenu. Cette considération n'a sans doute pas échappé au Conseil constitutionnel qui, après avoir examiné la question sous l'angle de l'égalité sans relever de violation des normes constitutionnelles, note qu'il est "loisible au législateur de modifier les dispositions législatives contestées pour préciser les délais » (consid. $\left.\mathrm{n}^{\circ} 14\right)$.

Pour l'heure, il appartient ainsi aux juges de vérifier la conformité du temps mis par la chambre de l'instruction pour statuer après renvoi à l'exigence de célérité constitutionnellement imposée. C'est donc au cas par cas, in concreto que revient aux juges le soin de se livrer à cette appréciation sans qu'un délai puisse être défini par avance. Ainsi, dans un arrêt rendu le 25 mars 2015, la Cour de cassation, répondant aux exigences posées par le Conseil constitutionnel le 29 janvier 2015, a jugé qu'une chambre de l'instruction, qui s'est prononcée le 27 novembre 2014 alors qu'elle n'a reçu le dossier, à la suite de l'arrêt de cas-sation, que le 10 novembre 2014, a statué dans de brefs délais sur la légalité de l'ordonnance de placement en détention provisoire (Cass. crim., 25 mars 2015 : pourvoi ${ }^{\circ} 14-88465$, inédit au bull.). Auparavant, elle avait pu juger conforme à l'exigence de célérité une décision rendue le 13 mars 2009 à la suite d'un arrêt de cassation du 21 janvier 2009, soit près de deux mois plus tard (Cass. crim., 8 juill. 2009 : pourvoi ${ }^{\circ}$ 09-82492).

3 - Droit de la défense. Audition d'une personne dans le cadre d'un mandat d'arrêt européen et d'une extradition

\section{Cass. crim., 6 janvier 2015, ${ }^{\circ}$ 14-87.893 (non-lieu à renvoi) - 21 jan-vier 2015, $n$ $\circ 14-87.380, n^{\circ} 14-87.042$ et $n^{\circ} 14-87.377$ (non-lieu à renvoi)}

Les droits de se taire et de ne pas contribuer à sa propre accusation en matière pénale sont désormais clairement consacrés par le code de procédure pénale au profit de nombreuses personnes tant lors de la phase policière que lors de l'instruction ou du jugement de l'affaire (v. en ce sens la réforme intervenue par l'effet de la loi n 2014-535 du 27 mai 2014 portant transposition de la directive 2012/13/UE du Parlement européen et du Conseil du 22 mai 2012 relative au droit à l'information dans le cadre des procédures pénales). 
Faut-il en déduire que ces droits doivent être généralisés à toute audition ? Les requérants dans les différents arrêts rendus par la Cour de cassation les 6 et 21 janvier dernier semblaient le penser puisqu'ils soutenaient que le fait, pour une personne recherchée dans le cadre d'un mandat d'arrêt européen devant la chambre de l'instruction (CPP, art. 695-30) ou dans le cadre d'une demande d'extradition (CPP, art. 696-14 et 695-15) d'être auditionnée sans avoir été au préalable informée de son droit de garder le silence serait contraire tant aux droits de la défense qu'au principe d'égalité devant les règles de procédure. Plus spécifiquement, sur ce second point, ils arguaient du fait que la personne qui comparait en personne devant la chambre de l'instruction dans le cadre de ces procédures est dans la même situation procédurale que la personne qui comparait devant une juridiction de jugement puisqu'elle est sous le coup d'une accusation au sens large, de telle sorte qu'aucune justification constitutionnellement recevable ne peut être apportée pour que l'une se voit notifier le droit de se taire alors que l'autre non.

Cette argumentation ne trouve toutefois pas grâce aux yeux de la Cour de cassation qui, dans ces différents arrêts, énonce de façon constante et claire que la procédure d'exécution d'un mandat d'arrêt européen, pas plus d'ailleurs que la procédure d'examen d'une demande d'extradition, ne conduit les juridictions françaises compétentes à recueillir des éléments d'accusation à l'égard de la personne concernée. Les auditions des personnes recherchées dans le cadre de ces procédures et pratiquées devant la chambre de l'instruction ne visent qu'à constater l'identité des personnes, à recevoir leurs observations sur la procédure dont elles font l'objet, et leur permettre de consentir ou non à leur remise. Le but de l'audition n'est donc en aucun cas de soumettre ces personnes à un inter-rogatoire sur les faits objet de la procédure. Dès lors, l'absence de notification du droit de se taire n'est pas contraire aux droits de la défense, et notamment au droit de la personne de ne pas contribuer à sa propre incrimination. Elle n'est pas davantage contraire au principe d'égalité. Pour ces raisons, la chambre criminelle refuse de transmettre les questions au Conseil constitutionnel.

À la fois clairs et rédigés en des termes similaires ces arrêts doivent être approuvés en ce que la notification du droit de se taire et de ne pas contribuer à sa propre accusation trouvent leur existence dans le risque que d'éventuelles révélations soient utilisées contre la personne pour établir son implication dans les faits. Or, il n'appartient aucunement à la chambre de l'instruction de statuer dans le cadre de ces procédures sur le bien-fondé d'une accusation en matière pénale. Elle ne fait pas, dans ces procédures, office de véritable juridiction appréciant l'implication d'une personne dans des faits ni même recueillant des éléments d'information sur l'accusation. Elle se borne à vérifier les conditions de légalité de la demande et à assurer le respect des droits fondamentaux de la personne réclamée. Seule la juridiction de l'État d'émission du mandat d'arrêt ou ayant formulé la demande d'extradition sera amenée à statuer sur le bien-fondé de l'accusation en matière pénale. (E. B.-G.) 
1 - Peines non manifestement disproportionnées

\section{Cons. const., 6 fév. 2015, $\mathrm{n}^{\circ}$ 2014-448 QPC ; Cons. const., 23 janv 2015, $\mathrm{n}^{\circ}$ 2014-439 QPC}

Dans l'espèce du 6 février 2015, l'auteur de la question prioritaire de constitutionnalité se fondait sur la démonstration développée au fond sur l'inconstitutionnalité des articles 222-22 et 222-22-1 du code pénal (v. supra) pour en conclure que la possibilité offerte par ces textes de retenir un même fait à la fois comme élément constitutif et circonstance aggravante de l'agression sexuelle rendait alors les peines encourues contraires aux principes de nécessité comme de proportionnalité découlant de l'article 8 de la Déclaration des droits de l'homme et du citoyen.

Le conseil éconduit l'argument par un raisonnement sibyllin : en disposant que la contrainte peut résulter de la différence d'âge existant entre une victime mineure et l'auteur des faits et de l'autorité de droit ou de fait que celui-ci exerce sur celle-ci alors que, par ailleurs, la peine encourue par l'auteur de ces infractions est aggravée lorsqu'il a, sur la victime, une autorité de droit ou de fait, les dispositions contestées n'instituent pas une sanction pénale qui mécon-naît les principes de nécessité et de proportionnalité des peines. On devine qu'ayant écarté le raisonnement sur le plan du fond du droit, le conseil en tire la conclusion qui s'impose : les dispositions contestées n'ayant pas vocation à faire du même élément à la fois un élément constitutif et une circonstance aggra-vante de la qualification d'agression sexuelle, les peines qu'ils induisent ne sont donc pas manifestement disproportionnées, le conseil se limitant à cette seule évaluation lorsqu'il a à apprécier la nécessité d'une peine (pour un exemple, Cons. const., 27 sept. $2013:$ déc. n ○ 2013-341 QPC).

Le conseil reprend cette motivation désormais classique dans une autre déci-sion extrêmement attendue (Cons. const., 23 janv. 2015, n $^{\circ} 2014-439$ QPC), elle aussi, en raison des événements tragiques du mois de janvier, au sujet de l'article 25 du code civil qui prévoit la déchéance de la nationalité française de celui qui a été condamné pour acte qualifié crime ou délit constituant une atteinte aux intérêts fondamentaux de la Nation ou pour un crime ou un délit constituant un acte de terrorisme, sauf si la déchéance aurait pour conséquence de la rendre apatride. Si le conseil conclut que, eu égard à sa nature - priver une personne de sa nationalité française - ce texte institue bien une sanction ayant le caractère d'une punition, celle-ci n'est en revanche pas manifestement disproportionnée car elle ne vise qu'un condamné pour des actes de terrorisme, particulièrement graves, et ne peut, de toute façon, le rendre apatride. (V.P.) 
Cons. const., 18 mars 2015, $n^{\circ}$ 2014-453/454 QPC et $n^{\circ}$ 2015-462 QPC : JCP G 2015, 368, obs. F. Sudre et 369, obs. J.-H. Robert, Dr. pén 2015, comm. à paraître

C'est une décision très attendue que le conseil a rendue le 18 mars 2015 puisqu'elle était censée résoudre l'ensemble des problèmes liés à la compatibilité des cumuls de poursuites et des sanctions susceptibles d'en résulter. En effet, pressée par les jurisprudences européennes, la Cour de cassation lui avait ren-voyé pas moins de trois questions prioritaires de constitutionnalité (Cass crim., 17 déc. 2014, $\mathrm{n}^{\circ}$ 14-90.042 et $\mathrm{n}^{\circ}$ 14-90.043 : Dr. pén. 2015, comm. 29 et Cass. crim., 29 janv. 2015, $\mathrm{n}^{\circ}$ 14-90.049) portant à la fois sur l'article 6 du code de procédure pénale et sur les articles L. 465-1, L. 466-1, L. 621-15-1, L. 621-16, L. 621-16-1 et L. 621-20-1 du code monétaire et financier, défi-nissant, notamment, l'infraction pénale de délit d'initié (art. L. 465-1) et le manquement d'initié (art. L. 621-15). L'article 6 du code de procédure pénale, qui mentionne la chose jugée parmi les causes d'extinction de l'action publique, était accusé de refuser de reconnaître l'autorité de la chose jugée aux décisions définitives de l'Autorité des Marchés Financiers (AMF), permettant de ce fait une double poursuite à la fois administrative et pénale conduisant à un possible cumul de sanctions, en contradiction avec le principe non bis in idem. Il était reproché aux dispositions du code monétaire et financier de permettre d'exercer des poursuites pénales, et le cas échéant de prononcer une condamnation, pour des faits ayant déjà fait l'objet d'une décision définitive de mise hors de cause rendue par la Commission des sanctions de l'Autorité des Marchés Financiers dans une même affaire, en méconnaissance, là aussi, du même principe.

L’influence européenne est avérée puisque, outre que le principe est protégé par l'article 4 du protocole $\mathrm{n}^{\circ} 7$ additionnel à la Convention de sauvegarde des droits de l'homme et des libertés fondamentales, la Cour de cassation fait expressément référence à l'arrêt rendu par la Cour européenne des droits de 1'homme, le 4 mars 2014 (CEDH, 2e sect., 4 mars 2014, nos 18640/10, 18647/10, 18663/10, 18668/10 et 18698/10, Grande Stevens et a. c/ Italie), comme étant susceptible de constituer un « changement de circonstances » de nature à faire évoluer la précédente position du Conseil constitutionnel (en faveur de la conformité de ces dispositions à la Constitution : Cons. const., 28 juill. 1989, n 89-260 DC. À noter que le Conseil d'État, dans une décision du 13 février 2015, a refusé de prendre en compte ce changement de circonstances et de renvoyer une question prioritaire de constitutionnalité au conseil en matière de discipline des vétéri-naires, arguant que les poursuites qui peuvent être engagées par les instances ordinales contre les vétérinaires en raison de manquements aux obligations défi-nies par le code rural et de la pêche maritime se rapportent à l'exercice de droits et obligations à caractère civil et non à une accusation en matière pénale : CE, 13 fév. 2015, 4e et 5e sous-sections réunies). De fait, il résulte de l'arrêt Grande Stevens c. Italie que constitue une violation du principe non bis in idem le fait de poursuivre pénalement des personnes déjà condamnées administrativement pour une même « infraction », les poursuites ayant pour origine des faits identiques à ceux qui ont fait l'objet de la première condamnation définitive (sur la notion de " même fait », v. CEDH, 10 févr. 2009, (GC), n 14939/03, Zolotoukhine c/ Russie). À cela s'ajoute que la Cour de justice de l'Union européenne avait déjà rendu deux arrêts dans lesquels elle affirme clairement que si le principe non bis in idem ne s'oppose pas à un cumul de sanctions administrative et pénale, c'est à la condition que la première n'ait pas un caractère pénal au sens de la Cour 
européenne des droits de 1'homme (CJUE, 26 févr. 2013, n C-617-10, Åklaga-ren c. Fransson ; CJUE, 5 juin 2012, $\mathrm{n}^{\circ} \mathrm{C}-489 / 10$, Bonda).

La position des juridictions françaises (par exemple, Cass. crim., 25 juin 2014, $\mathrm{n}^{\circ}$ 13-87.692 : Dr. pén. 20144, comm. 122 ; Rapp. Cass. crim., 23 juill. 2014, $\mathrm{n}^{\circ}$ 14-80.428 : JurisData $\mathrm{n}^{\circ}$ 2014-018435 ; Cah. Cons. const. 2014, $\mathrm{n}^{\circ} 46$ ), initiée par le Conseil constitutionnel (pour un exemple, Cons. const., 27 sept. 2013, déc. $\mathrm{n}^{\circ}$ 2013-341 QPC), aux termes de laquelle un cumul de sanctions était possible si le total des sanctions n'excédait pas le maximum de la sanction le plus élevé, s'en trouvait menacée de sorte qu'il devenait urgent que le conseil se prononce.

Celui-ci rend pourtant une solution en demi-teinte, principalement au sujet des dispositions du code monétaire et financier qui, même si elle se comprend, n'en demeure pas moins insatisfaisante (l'article 6 du code de procédure pénale est quant à lui déclaré conforme dans une réponse lapidaire : «s'il résulte de [ses dispositions] qu'une décision définitive d'une autorité administrative pronon-çant une sanction ayant le caractère d'une punition ne constitue pas une cause d'extinction de l'action publique, elles ne méconnaissent en ellesmêmes aucun autre droit ou liberté que la Constitution garantit ; les mots "la chose jugée" figurant au premier alinéa de l'article 6 du code de procédure pénale doivent donc être déclarés conformes à la Constitution »).

Pour se déterminer, le conseil énumère quatre critères destinés, s'ils sont réunis, à interdire le cumul de sanctions : d'abord, les articles L. 465-1 et L. 621-15 du code monétaire et financier tendent à réprimer les mêmes faits et protègent, ensuite et par conséquent, les mêmes intérêts sociaux (l'ordre public écono-mique). De plus, les faits incriminés sont susceptibles de faire l'objet de sanctions « qui ne sont pas de nature différente », ce qui est une référence non dissimulée à la jurisprudence Åklagaren et Bonda précitée (voir déjà Cons. const., 24 oct. 2014, $\mathrm{n}^{\circ}$ 2014-423 QPC : Dr. pén. 2015, comm. 14). Enfin, le conseil prend sur lui d'ajouter un quatrième critère pour prohiber le cumul des poursuites comme des sanctions: ces dernières, tant pour le délit (pénal) d'initié que pour le manquement (boursier) d'initié sont prononcées par des juges appartenant au même ordre de juridiction (en effet, l'appel contre les décisions individuelles de l'AMF est examiné par le juge judiciaire).

Le conseil en tire donc la conclusion que les dispositions litigieuses contredi-sent le principe de la légalité des délits et des peines mais abroge le seul texte incriminant le délit d'initié, avec effet différé au 1er septembre 2016 pour éviter toutes difficultés liées à une disparition immédiate du texte. Entre-temps, il interdit que des poursuites soient engagées ou continuées devant le juge pénal pour délit d'initié si l'AMF a déjà rendu une décision ayant autorité de la chose jugée sur le fondement du manquement d'initié et inversement, se mettant en conformité avec la solution d'espèce de l'arrêt Grande Stevens : les doubles pour-suites sont désormais interdites en matière boursière.

Toutefois, au-delà de cette solution immédiate, on perçoit en filigrane que le dessein du conseil, lorsqu'il ajoute le critère de l'unicité de juridiction, est à la fois de sauvegarder la conception française du cumul pour que celui-ci ne soit prohibé que dans un certain nombre de contentieux - dont le contentieux boursier - et de limiter les conséquences de la jurisprudence Grande Stevens qui, précisément parce qu'elle ne limite pas l'interdiction du cumul aux sanc-tions prononcées par des juridictions du même ordre, pose au contraire une interdiction très générale. 
Mais il n'en demeure pas moins que, de ce fait, il se place en contradiction de principe avec les deux cours européennes (voir aussi la solution de la Cour de cassation qui casse l'arrêt d'une cour d'appel ayant appliqué la solution de l'arrêt Grande Stevens à un notaire déjà condamné pénalement à une interdiction d'exercice temporaire et lui ayant reconnu expressément le droit de se prévaloir du principe non bis in idem pour éviter d'être condamné disciplinairement à la destitution, au motif lapidaire que les sanctions ne sont pas de même nature (mais que sont, alors, des sanctions de même nature ?) : Cass. civ. $1^{\text {re }}, 9$ avr. 2015, n $^{\circ} 14-50012$, à paraître au bull.). Pour illustration de cette divergence, selon le conseil, le contentieux fiscal échappe à l'interdiction du cumul puisqu'il relève de la juridiction administra-tive (les sanctions ne seront donc pas prononcées par des juridictions relevant du même ordre), alors que la Cour européenne des droits de l'homme consi-dère qu'il s'agit de matière pénale (CEDH, Bendenoun c. France, 24 fév. 1994, n 12547/86) de sorte que par application des décisions Åklagaren et Bonda de la Cour de justice de l'Union européenne, il ne saurait y avoir de cumul de sanctions puisque les sanctions fiscales susceptibles d'être infligées relèvent de la matière pénale. De plus, la décision constitutionnelle d'abroger la qualification de délit d'initié pose question lorsqu'on la rapproche des directives 2003/6/CE du Parlement européen et du Conseil du 28 janvier 2003 et 2014/57/UE du Parlement et du Conseil du 16 avril 2014: la première se prononce en faveur d'une double répression administrative et pénale en matière boursière, tandis que la seconde incite les États à incriminer la divulgation illicite d'informations privilégiées. En outre, la décision Grande Stevens c. Italie ne s'oppose pas à une double qualification des faits mais simplement à leur double poursuite pour éviter le cumul de sanctions qui s'ensuivrait.

Cependant, cette décision décevante s'explique parce qu'en abrogeant la qualifi-cation de délit d'initié, le conseil répond à une question procédurale (l'interpré-tation du principe non bis in idem) par une solution de fond: 1'article L. 465-1 du code monétaire et financier n'est pas conforme à la Constitution, non pas parce qu'il ignore le principe non bis in idem, mais parce qu'il méconnaît le principe de nécessité des délits et des peines. En définitive, coincé entre les exigences européennes et la nécessité de préserver autant que faire se peut la conception française du cumul de sanctions qu'il a lui-même peaufinée, le conseil indique qu'il ne lui appartient pas de régler un problème dont l'ampleur dépasse sa compétence et qui entre tout naturellement dans celle du législateur. (V.P.)

\section{3 - Réduction de peine}

\section{CE (6e et 1re sous-sections réunies), 25 mars 2015 , req. $\mathrm{n}^{\circ} 374401$}

Par un arrêt en date du 25 mars 2015, le Conseil d'État a eu à connaître d'une question prioritaire de constitutionnalité portant sur l'article 721 du code de procédure pénale relatif aux crédits de réduction de peine, laquelle est l'occasion de revenir sur la nature des réductions de peine et d'entériner la solution récemment dégagée à ce sujet par le Conseil constitutionnel. Était plus précisément en cause l'article 721, alinéa 5 du code de procédure pénale qui dispose qu'en cas de nouvelle condamnation à une peine privative de liberté pour un crime ou un délit commis par le condamné après sa libération pendant une période égale à la durée de la réduction, la juridiction de jugement peut ordonner le retrait 
de tout ou partie de cette réduction de peine et la mise à exécution de l'emprisonnement correspon-dant, qui n'est pas confondu avec celui résultant de la nouvelle condamnation. En l'occurrence, le requérant soutenait que cet alinéa était contraire à la fois au principe $\mathrm{Ne}$ bis in idem et au principe de nécessité et de pro-portionnalité des peines découlant de l'article 8 de la Déclaration des droits de l'homme et du citoyen. À en croire cette question, ce serait condamner deux fois une personne à raison de mêmes faits que de prononcer contre elle d'une part, une sanction en réponse à la nouvelle infraction cause de révocation du crédit et d'autre part, un retrait du crédit de réduction de peine.

Se fondant exclusivement sur la nature de ce retrait, le Conseil d'État écarte l'argument et dit n'y avoir lieu à renvoi de la question au Conseil dès lors que le retrait ne constitue ni une peine ni une sanction ayant le caractère d'une punition.

En ce sens, le Conseil d’État reprend la qualification donnée par le Conseil constitutionnel au retrait du crédit dans une décision du 11 juillet 2014 (Cons. const., 11 juill. 2014, déc. ${ }^{\circ}$ 2014-408, QPC, v. notre commentaire Les Nouveaux cahiers du Conseil constitutionnel, 2014, $\mathrm{n}^{\circ}$ 46). Cette décision du Conseil constitutionnel qui avait été rendue à la suite de la transmission d'une question par le Conseil d'État montre à quel point cette juridiction administrative aura œuvré à dégager la nature et le régime juridique des réductions de peine. Dans le même sens, on peut faire état d'une autre décision rendue sur la même période par le Conseil d'État le 18 février 2015, portant non pas sur une question prioritaire de constitutionnalité, mais ren-due dans le cadre d'un recours en annulation pour excès de pouvoir des articles D. 115-7 à D. 115-12 du code de procédure pénale. Par cette décision, le Conseil d’État juge en effet que les termes de l'article 721 du code de procédure pénale définissent avec suffisamment de précision et de clarté ce qu'il convient d'entendre par « mauvaise conduite en détention » dans la mesure où les obligations et interdictions qui s'imposent aux personnes détenues sont elles-mêmes définies par les dispositions du titre 2 du Livre 5 du code de procédure pénale, par la loi pénitentiaire du 24 novembre 2009 et par le règlement intérieur type de chaque établissement pénitentiaire fixé en vertu de l'article 728 du code de procédure pénale (CE, 18 févr. 2015, req. $n^{\circ}$ 375765). (E. B.-G.)

Revue doctrinale

\title{
Articles relatifs aux décisions du Conseil constitutionnel
}

\author{
25 octobre 2013
}

\section{3-350 QPC}

Commune du Pré- Saint- Gervais [Mise en œuvre de l'action publique en cas d'injure ou de diffamation publique envers un corps constitué] - Bellescize, Diane de. « QPC, loi du 29 juillet 1881, articles 47 et 48 », Constitutions. Revue de droit constitutionnel appliqué, octobre- décembre 2014, n 2014-4, p. 503-504. 


\section{4-403 QPC}

\section{Laurent L. [Caducité de l'appel de l'accusé en fuite]}

- Teissedre, Jean-Charles. «De quelques aspects insoupçonnés d'une QPC. À propos de l'abrogation de l'alinéa 5 de l'article 380-11 du Code de procédure pénale », Droit pénal, mars 2015, n 3 , p. 10-12.

\section{6 septembre 2014}

\section{4-416 QPC}

Association France Nature Environnement [Transaction pénale sur l'action publique en matière environnementale]

- Guerin, Martin. «La transaction pénale environnementale constitutionnelle », Énergie environnement - infrastructures : actualité, pratiques et enjeux, janvier $2015, \mathrm{n}^{\circ} 1$, p. 43-46.

- Robert, Jacques- Henri. « Transaction en matière d'environnement », Revue de science criminelle et de droit pénal comparé, octobre- décembre 2014, n 4 , p. 785-788.

\section{4-418 QPC}

\section{8 octobre 2014}

\section{Société SGI [Amende pour contribution à l'obtention, par un tiers, d'un avantage} fiscal indu]

- Le Bot, Olivier. «Le pouvoir de sanction de l'administration fiscale », Constitutions. Revue de droit constitutionnel appliqué, octobre- décembre 2014, n 2014-4, p. 489-491.

\section{4-420/421 QPC}

\section{9 octobre 2014}

M. Maurice L. et autre [Prolongation exceptionnelle de la garde à vue pour des faits d'escroquerie en bande organisée]

- Gallois, Alexandre. « Escroquerie en bande organisée : inconstitutionnalité de la garde à vue de quatre jours », Bulletin Lamy droit pénal des affaires, novembre 2014, n 144 , p. 1-6.

- Perrier, Jean- Baptiste. « Le Conseil constitutionnel, l'escroquerie en bande organisée et la garde à vue : l'abstraction de l'inconstitutionnalité », Actualité juridique. Pénal, décembre 2014, n 12 , p. 574-577.

\section{4-423 QPC}

\section{4 octobre 2014}

M. Stéphane R. et autres [Cour de discipline budgétaire et financière] - Peltier, Virginie. «L'avenir du principe non bis in idem et ses répercussions sur les cumuls de sanctions », Droit pénal, février 2015, n 2, p. 51-52.

- Peltier, Virginie. «Infléchissement de la validation constitutionnelle des cumuls de sanctions pénale et administrative », Droit pénal, janvier 2015, n 1, p. 36-37. - RoblotTroizier, Agnès. « Les principes constitutionnels du procès équitable ne sont pas applicables à l'enquête administrative préalable à la saisine de la Cour de discipline budgétaire et financière » in : Chronique de jurisprudence - Droit administratif et droit constitutionnel. Revue française de droit administratif, novembre- décembre 2014, ${ }^{\circ} 6$, p. 1218-1223. 


\section{4-428 QPC}

M. Nadav B. [Report de l'intervention de l'avocat au cours de la garde à vue en matière de délinquance ou de criminalité organisées] - Perrier, Jean-Baptiste. « Criminalité organisée : constitutionnalité du report de l'intervention de l'avocat lors de la garde à vue », Actualité juridique. Pénal, février 2015, n², p. 100-101.

\section{3 janvier 2015}

\section{4-439 QPC}

\section{Ahmed S. [Déchéance de nationalité]}

- Spinosi, Patrice. « Le Conseil constitutionnel encadre le pouvoir du législa-teur pour déchoir de leur nationalité les terroristes », La Semaine juridique. Édi-tion générale, 2 février 2015, n 5, p. 190.

\section{Articles thématiques}

Droit pénal

- Botton, Antoine. « Le droit à un recours juridictionnel effectif dans le cadre de l'enquête pénale », Constitutions. Revue de droit constitutionnel appliqué, octobre- décembre 2014, $\mathrm{n}^{\circ}$ 2014-4, p. 471-475.

- Hilger, Geoffroy. « La fonction renouvelée de l'interprétation conforme en droit pénal », Revue de la recherche juridique, droit prospectif, février 2015, n 2014-2, p. 819-849.

- Lamy, Bertrand de. «Le Code pénal : consolidé ou fragilisé par le droit constitutionnel ? » in Le nouveau code pénal : 20 ans après ; état des questions. Paris, LGDJ, 2014, p. 21-36. - Lasserre Capdeville, Jérôme. « L’article 225-3- 1 du code pénal, régissant le testing, est conforme aux droits de la défense et au droit à un procès équitable.[Cass. crim. 4 février 2015, n 14-90048] », Actualité juridique. Pénal, mars 2015, n 3, p. 139-141.

- Le Monnier de Gouville, Pauline. « La juridictionnalisation de 1'enquête pénale », Les Cahiers de la justice, mars 2015, n²015/1, p. 131-139.

- Mésa, Rodolphe. « Autorité de la chose jugée, cumul de qualifications et de sanctions punitives : retour sur la règle non bis in idem. [Cass. crim., 9 déc. 2014, n ${ }^{\circ}$ 13-85937; Cass. crim., 1 er déc. 2014, $\mathrm{n}^{\circ}$ 14-80230 ; Cass. crim., 17 déc. 2014 n ${ }^{\circ}$ 14-90042, Cass. crim., 17 déc. 2014, n 14-90043] », La Gazette du Palais, 23 et 24 janvier 2015, n²3-24, p. 17-20.

- Peltier, Virginie. « L'avenir du principe non bis in idem et ses répercussions sur les cumuls de sanctions », Droit pénal, février 2015, n² 2, p. 51-52.

- Robert, Jacques- Henri. « Le jeu du mistigri judiciaro- constitutionnel. [Cass. crim., 17 déc. 2014, n 14-90.042] », Droit pénal, février 2015, n² 2, p. 43-44. 